\title{
Tunable Single-Photon Ionization TOF Mass Spectrometry Using Laser-Produced Plasma as the Table-Top VUV Light Source
}

\author{
Tonia M. Di Palma, Maria V. Prati, and Antonio Borghese \\ Istituto Motori-CNR, Naples, Italy
}

Here we report on a laser plasma-based tunable VUV photoionization time-of-flight (TOF) mass spectrometer conceived mainly to study complex gaseous mixtures. Ionizing photons at tunable vacuum UV (VUV) wavelengths are generated by a gas-target laser-produced plasma, spectrally dispersed in the range 100-160 nm and efficiently focused onto a sample molecular beam. As a test case, we studied the exhaust gas of a four-stroke moped, a typical example of a complex gaseous mixture. Due to the VUV "soft" ionization, the mass spectra are less congested and more easily interpretable. Substituted benzene derivatives are found to give the most intense signals. Several aliphatic hydrocarbons are also detected. The use of tunable VUV radiation allowed the investigation of the contribution of isomers in the mass spectrum from the onset and shape of the photoionization efficiency spectra. Semiquantitative analysis was performed using known literature data detailing the photoionization cross sections. Our findings suggest that using combined data on the mass/photoionization efficiency spectra may be very helpful for a comprehensive analysis of complex gaseous mixtures. (J Am Soc Mass Spectrom 2009, 20, 2192-2198) (c) 2009 American Society for Mass Spectrometry

I n the analysis of complex gaseous mixtures, a key concern is the ability to simultaneously, sensitively, and selectively quantify many species using techniques that have the potential for integration with table-top instrumentation.

Most approaches use time-of-flight mass spectrometry (TOF-MS) with either electron impact (EI) or chemical ionization. Each of these methods possesses its own drawbacks, such as the fragmentation of compounds (EI) or the inability to distinguish between isomers. Laser-based spectroscopic techniques (e.g., resonanceenhanced multiphoton ionization or REMPI), on the other hand, are extremely selective and sensitive for specific compounds, but lack broader applicability. In particular, although REMPI is useful for the analysis of many aromatic compounds, it is unable to detect most aliphatic species through simple single-wavelength excitation schemes. Rather, complex multi-color approaches requiring multiple lasers must generally be used for detection of non-aromatic compounds. Using whatever approach, REMPI can only be effectively applied to detect compounds whose electronic states are well known.

An alternative approach to meet the requirements mentioned above is the combination of TOF-MS with VUV-tunable single-photon ionization between 12 and $6 \mathrm{eV}(100-200 \mathrm{~nm})$. This region of the UV spectrum corresponds to energies matching the ionization poten-

Address reprint requests to Dr. T. M. Di Palma, Istituto Motori - CNR, Via Marconi 8, 80125 Napoli, Italy. E-mail: t.dipalma@im.cnr.it tials (IPs) of many organic compounds. In most cases, the coupling of tunable VUV single-photon ionization to TOF-MS simplifies interpretation of mass spectra because tuning the photon energy closely around the ionization threshold generally results in nondissociative photoionization, particularly when compounds are cooled in a molecular beam [1,2]. Such an approach can allow acquisition of the photo-ionization efficiency (PIE) spectrum for each compound, thereby providing the potential to discriminate isomers with different IPs. The method has proven to be very powerful in many experiments using tunable VUV synchrotron light sources at large-scale facilities [3-6]. Unfortunately, while synchrotron light sources can provide the tunability and VUV photons needed for "soft" photoionization, they are obviously unfit for on-site and in situ application.

Tunable VUV laser sources such as optical parametric oscillators (OPOs) are particularly appropriate for mixture analysis on a laboratory scale [7]. Several drawbacks to the use of such tunable laser systems are their cost, complexity, and lack of portability.

Recently, we have carried out characterization studies on laser-produced plasmas (LPPs) [8] and have proposed an LPP-based VUV light source suitable for application of tunable single-photon ionization to TOFMS [9]. Our findings indicated that LPPs emit a wide continuum of radiation in the useful wavelength range of 100-200 nm. Such a wavelength range is not achievable with traditional laser sources, and the photon 
fluxes produced by the LPPs during emission are much greater than those of glow-discharge lamps [9].

Here we report on the performance of a new apparatus using VUV broadband radiation from a gas-target LPP as an ionization source coupled to a TOF mass spectrometer and show proof-of-concept results for the gas-phase analysis of volatile organic compounds (VOCs) emitted in the exhaust of a four-stroke moped, an exemplary case of a complex gaseous mixture. The VOCs emitted from vehicles result mainly from incomplete combustion. They act as primary toxic pollutants and are involved in the secondary formation of organic aerosols. Moreover, they are precursors for ozone formation. Mopeds are two-wheeled motor vehicles with engines having a stroke volume smaller than $50 \mathrm{~cm}^{3}$ and maximum speeds up to $45 \mathrm{~km} / \mathrm{h}$. As their emission standards are less stringent than those of passenger cars and due to their large use in urban areas, emissions of two-wheelers have become increasingly relevant [1012]. The concentrations of VOCs in the exhaust are usually measured with traditional gas chromatographic methods [13]. Different analysis methods for monitoring the exhaust composition have proposed the use of TOF mass spectrometry with VUV single photon ionization at a fixed wavelength produced with coherent [14] or incoherent sources [15]. However, the use of an ionization source with a fixed wavelength only allows partial speciation of the compounds due to the presence of isomers. Use of a wavelength-tunable ionization source has the potential to add useful spectroscopic information to the mass spectra by improving the ability to differentiate between isomers in the gas mixture.

\section{Experimental}

An experimental schematic is shown in Figure 1. The tunable SPI-TOF mass spectrometer consists of two main vacuum chambers, each evacuated by its own turbomolecular pump (1000 litres/s). The VUV light source, based on a laser-produced plasma, is housed in the chamber sketched on the right side of Figure 1.

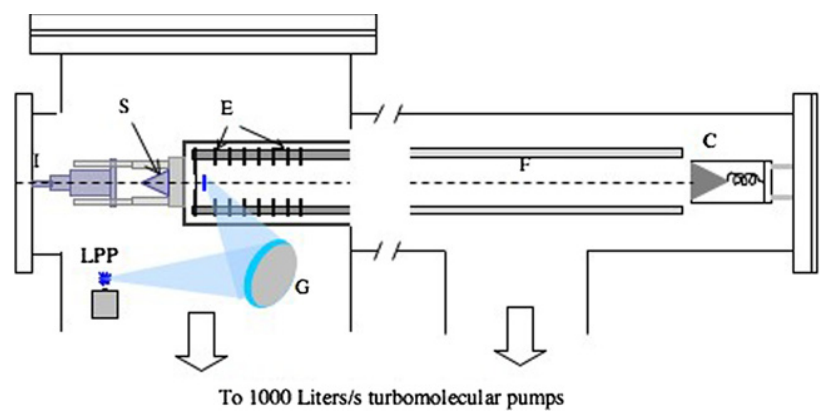

Figure 1. A schematic of the TOF mass spectrometer equipped with the LPP source: $\mathrm{I}=$ gas sample inlet, $\mathrm{S}=$ skimmer, $\mathrm{E}=$ extraction and focusing electrodes, $\mathrm{F}=900 \mathrm{~mm}$ free flight region, $\mathrm{C}=$ channeltron, $\mathrm{G}=$ grating. The detailed layout of the VUV source is reported in references $[8,9]$.
Though a description of the VUV source experimental set-up has been given elsewhere [8,9], it is worth providing full detail here for the sake of completeness. The LPP is produced by focusing an Nd-YAG laser (1064 nm, 10 ns, $200 \mathrm{~mJ}, 3 \mathrm{~Hz}$ ) on a supersonic Xe gas jet with an A/R-coated plano-convex lens (focal length = $10 \mathrm{~cm}$ ). The supersonic jet is emitted by a commercial solenoid valve (model 99, Parker Hannifin Corporation, General Valve Division, Fairfield, NJ, USA) at a back pressure of 10 bar with an aperture time of $400 \mu \mathrm{s}$. The emitted radiation is collected, spectrally dispersed by a concave flat-field diffraction grating (70 $\mathrm{mm}$ diameter, $210 \mathrm{~mm}$ focal length, $8 \mathrm{~nm} / \mathrm{mm}$ dispersion, blazed at $220 \mathrm{~nm}$ ), and focused in the center of the vacuum chamber. We found that the emission spectrum of the xenon plasma is mostly continuous in the 100-200 nm wavelength range and, in the used embedded configuration, VUV photon fluxes exceeding $10^{12}$ photons $/ \mathrm{cm}^{2} \times$ nm $\times$ pulse (with $2 \%$ BW @150 nm) have been measured at the grating focus in the first $20 \mathrm{~ns}$ following the laser onset [9].

In the same chamber, a second pulsed valve is used for the gas sample intake. A gaseous mixture at 1 bar is sent to the intake valve, which ejects a supersonic jet toward a $0.4 \mathrm{~mm}$ conical skimmer. A molecular beam coaxial to the TOF tube and crossing the VUV optical axis at $90^{\circ}$ is then selected. The skimmer is anchored directly to the wall of an inner chamber (i.d. $60 \mathrm{~mm}$ ) housing the extraction fields (see Figure 1) of a TOF mass spectrometer such that the distance between the valve nozzle and the ionization region is minimized to $60 \mathrm{~mm}$. The inner chamber is equipped with $10 \times 30$ $\mathrm{mm}^{2} \mathrm{MgF}_{2}$ windows that allow the VUV photons to reach the molecular beam while preventing undesired valve-injected gases from reaching the ionization and flight zone.

The mass spectrometer is a home-made linear TOF equipped with extraction/acceleration electrodes and seven cascaded focusing electrodes. A pulsed electrical field $\left(\mathrm{E}_{\text {ext }}=200 \mathrm{~V} / \mathrm{cm}, \Delta \mathrm{t}=3 \mu \mathrm{s}\right)$ is used to extract the ions from the region in which the sample interacts with the VUV radiation. The onset of the extraction potential is delayed by $400 \mathrm{~ns}$ with respect to the emission of the laser spark. Following the acceleration electrode $\left(\mathrm{E}_{\mathrm{acc}}=\right.$ $200 \mathrm{~V} / \mathrm{cm}$ ), an additional seven focusing electrodes are connected with a resistor divider such that the electric fields are $257 \mathrm{~V} / \mathrm{cm}$. The TOF free-field length is 960 $\mathrm{mm}$. The spectrometer is also equipped with ion optics (x-y deflection plates and an Einzel lens) that allow control of the ion collection and focusing on the Channeltron detector (model 4821G, Burle Industries, Lancaster, PA, USA).

In our configuration, the spectral resolution achievable for the photon energy is a function of the grating dispersion $(8 \mathrm{~nm} / \mathrm{mm})$ as well as the transverse sizes of the LPP $[8,9]$ and of the molecular beam. Its overall value was determined by recording the mass spectral intensity while scanning the 0th order reflection of the grating, i.e., the LPP shape, across the molecular beam. 
We measured a line width of about $0.8 \mathrm{~mm}$ (FWHM), corresponding to a wavelength (resp. energy) resolution of about $\pm 3 \mathrm{~nm}$ (resp. $\pm 0.2 \mathrm{eV} @ 9 \mathrm{eV}$ ).

A stepping motor is used for spectral scanning and a custom-made LabVIEW program driving a DAQ card (PCI-5152, National Instruments Corporations, Austin, TX, USA) for data acquisition. Typically, 1000 shots are accumulated to give one mass spectrum at each wavelength: the higher the laser repletion rate, the shorter the acquisition time. Mass spectra are sequentially acquired at $0.8 \mathrm{~nm}$ steps over the spectral band 100-160 $\mathrm{nm}$. All the data presented here have been corrected for the VUV intensity of the LPP.

Undiluted raw gas was sampled at the exhaust of a Vespa Piaggio moped (50 $\mathrm{cm}^{3}$, 4-stroke, 4 valves, complying with Euro 2 emission standards, Piaggio, Pontedera, PI, Italy) equipped with a passive (no air pump involved) secondary air injection system (SAI) downstream of the exhaust valves. The gas was spilled downstream from the SAI and upstream from the catalytic converter by filling a tank, at idle conditions, during the cold-start period of a driving cycle performed on a two-wheeler chassis dynamometer with electrical simulation of road load and inertia. Commercial gasoline was utilized, and its analysis is reported in Table 1. The exhaust gas sample was diluted with helium (dilution ratio $1: 10$ ) and sent to the inlet gas system of the apparatus. Naphthalene $(\mathrm{M}=128 \mathrm{u}, \mathrm{IP}=$ $8.14 \mathrm{eV}$, vapor pressure $\cong 10 \mathrm{~Pa} @ 25^{\circ} \mathrm{C}$ ) was chosen for mass and wavelength calibration and as an internal standard for the semiquantitative analysis. Therefore, all the measurements were performed by flowing the gaseous mixtures over naphthalene flakes at $25^{\circ} \mathrm{C}$.

\section{Results and Discussion}

\section{Detection Limits}

A drawback of (incoherent) VUV photoionization is stray light, which affects the mass spectra with significant noise. Signals corresponding to compounds with ionization potentials higher than $10 \mathrm{eV}$ (mainly bulk compounds such as helium, water, nitrogen, and oxygen) are detected. They are due to secondary ionization following the electron emission from surfaces irradiated by VUV light. Improvements are obtained with a delayed extraction field and a small negative bias $(-2 \mathrm{~V})$

Table 1. Gasoline specification provided by ENI S.p.A., Divisione Refining and Marketing, Laboratory di San Donato Milanese (MI), Italy

\begin{tabular}{cr}
\hline Gasoline composition & (vol\%) \\
\hline \hline Benzene & 0.6 \\
Aromatics & 33.3 \\
Olefins & 8.8 \\
Paraffins & 49.8 \\
Oxigenated & 8.1 \\
MTBE & 8.0 \\
\hline
\end{tabular}

on the repeller electrode. By delaying the extraction by $\sim 400 \mathrm{~ns}$, the helium mass peak disappears and signals from the other bulk compounds are greatly reduced. Also, the signal/noise ratio of the full mass spectrum is increased as a result of better focusing of the peaks (time-lag focusing).

Figure 2 shows a mass spectrum (5000 acquisition events) obtained by flowing 1 bar of helium in a reservoir with naphthalene, with $10.5 \mathrm{eV}$ ionization energy. The two detected peaks at $\mathrm{m} / \mathrm{z} 128$ and 129 correspond to the two most abundant isotopes of naphthalene. From the figure, the mass resolution is $\mathrm{m} / \Delta \mathrm{m} \approx 420$ at $m / z$ 128. The inset in Figure 2 shows an enlarged view of the vertical scale and allows estimation of the detection limit. By defining the detection limit (DL) as the concentration of a compound that gives a signal twice the background noise, it can be expressed by DL = $2 \sigma \mathrm{c} / \mathrm{h}$ [11], were $\sigma$ is the amplitude of the noise and $\mathrm{c}$ and $\mathrm{h}$ are the naphthalene concentration ( 100 ppm @ $\left.25^{\circ} \mathrm{C}\right)$ and ion peak area, respectively. Using these values, the measured detection sensitivity for naphthalene at an ionization energy of $10.5 \mathrm{eV}$ (Figure 2) is $\sim 1$ ppmv.

\section{Isomer Identification}

During a cold start, most of the gasoline components are expected to be found in the exhaust. Gasoline is a hydrocarbon mixture (paraffins, olefins, and aromatics) blended with a significant percentage of branched ethers (mainly methyl tert-butyl ether or MTBE) used as anti-knock agents (see Table 1). The aromatics primarily consist of methylated derivatives of benzene (toluene, xylenes) while the benzene content itself is kept low ( $<1 \%$ vol. in commercial EU EN228 gasoline) due to its high toxicity.

Mass spectra of moped exhaust as a function of photon energy are shown in Figure 3. In addition to the naphthalene peak at $m / z 128$, the most intense peaks are at $m / z 92,106,120$, and 134 . They are assigned to toluene $(m / z 92)$ and substituted derivatives of benzene. The peak at $m / z 74$ is due to diethyl ether, which is a contaminant from previous measurements. As shown in Figure 3, the intensity of these peaks decreases gradually with decreasing photon energy, and they disappear in the mass spectrum when the photon energy becomes lower than their ionization potential. The intensity of the peaks detected at $\mathrm{m} / \mathrm{z} 32$ and 44 does not depend on the ionizing photon energy. These peaks are assigned to oxygen and carbon dioxide and are thought to result from secondary ionization processes.

Figure 4 shows the PIE spectra for some of the most abundant compounds detected in the moped exhaust. These PIE spectra were obtained by integrating the area of each mass peak as a function of photon energy. The energy scale in Figure 4 was calibrated using the onset of the PIE spectrum of the naphthalene and diethyl ether ions. The arrows indicate the photon energy corresponding to the ionization potentials of the com- 


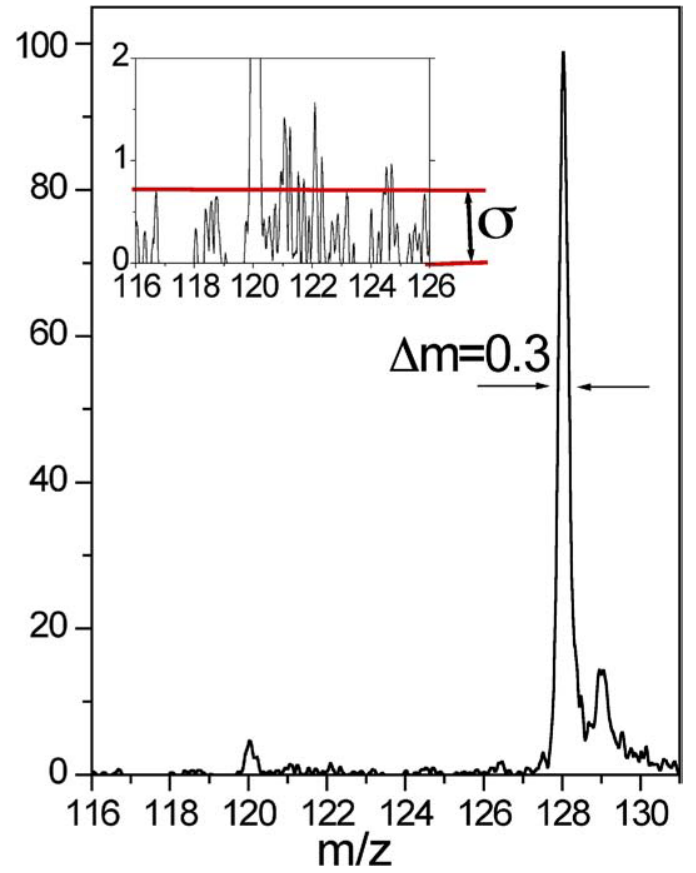

Figure 2. Mass spectrum of naphthalene acquired for 5000 excitation events using $10.5 \mathrm{eV}$ photon energy. The inset shows an enlarged view of the spectrum for evaluation of the standard deviation of the noise.

pounds shown at the top of the arrows. These values were acquired from the NIST database [16].

The PIE spectrum of toluene, reported in Figure 4a, shows an onset at $8.8 \mathrm{eV}$ and a slow rise up to $10 \mathrm{eV}$, in

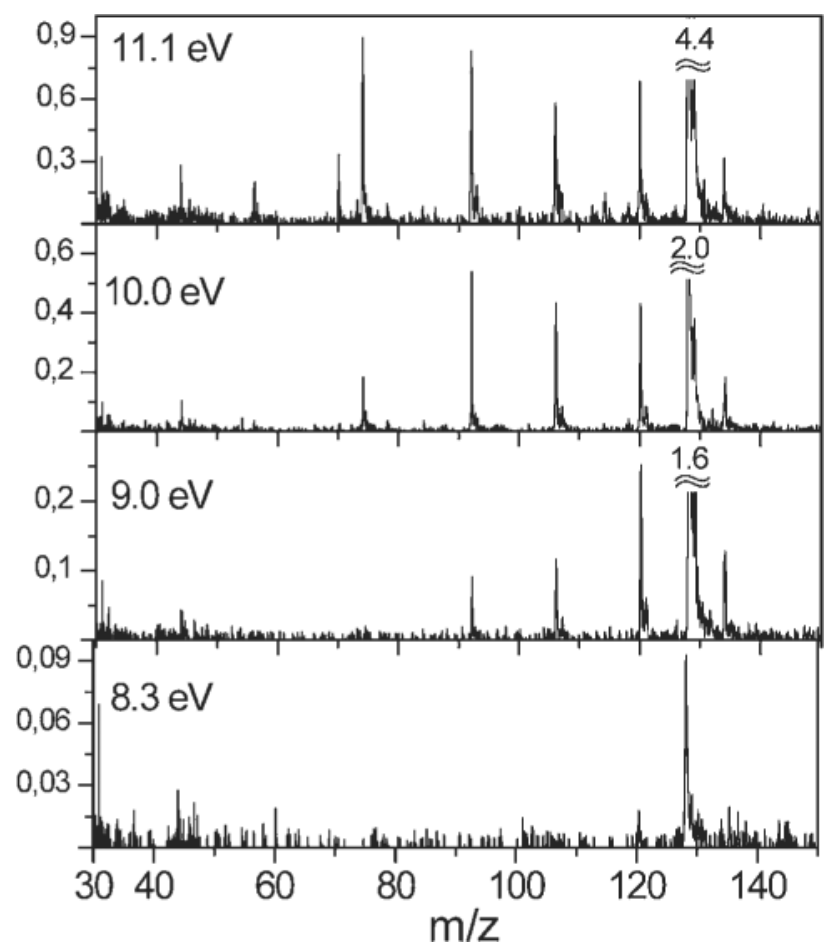

Figure 3. Mass spectra (1000 acquisition events) at different ionizing photon energies. The intensity value of the scaled-off peaks is reported near the peak top.
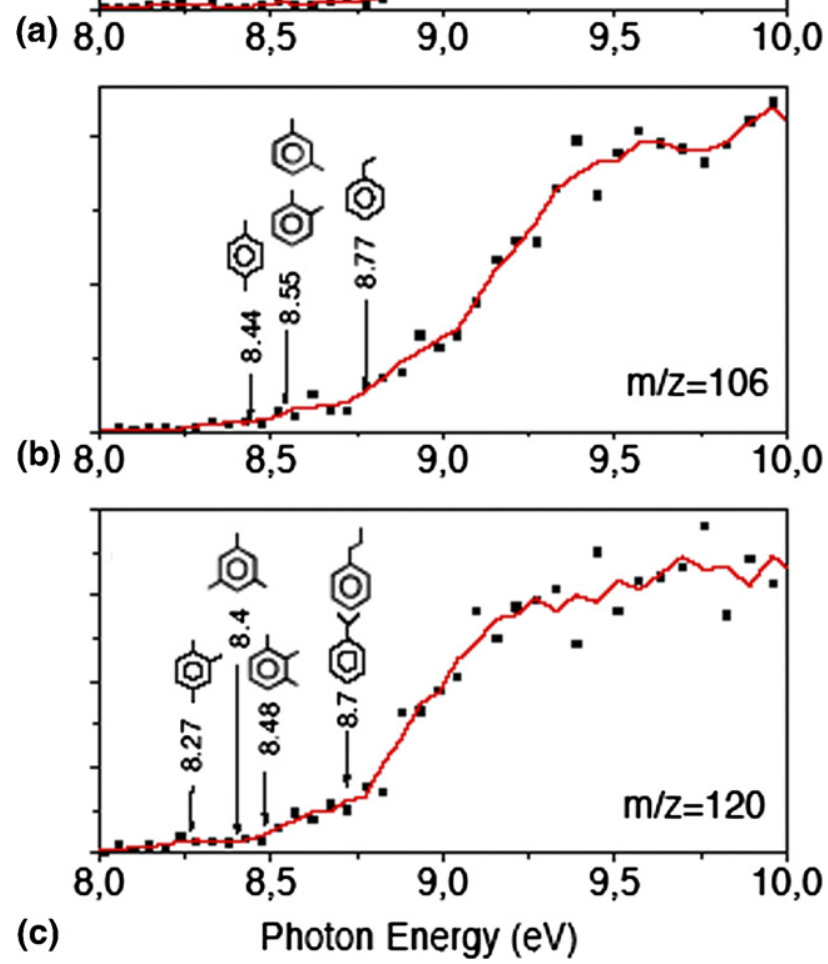

Figure 4. Photoionization efficiency spectra of the compounds $\mathrm{C}_{7} \mathrm{H}_{8}(\mathbf{a}), \mathrm{C}_{8} \mathrm{H}_{10}(\mathbf{b})$, and $\mathrm{C}_{9} \mathrm{H}_{12}$ (c). The lines are 3 point adjacent averaged smoothings. The arrows indicate the ionization potential of the compounds drawn above the arrows, as reported in the NIST database [16].

agreement with literature data (see Figure 5 of [6] and Figure 2 of [17]). Figure $4 \mathrm{~b}$ shows the PIE spectrum of $\mathrm{C}_{8} \mathrm{H}_{10}(106 \mathrm{u})$, interpreted as the contribution of four isomers of aromatic compounds, $\mathrm{m}$, $\mathrm{p}$, o-dimethylbenzene, and ethylbenzene, at different concentrations. Despite the low-energy resolution, the onset of this PIE curve seems closer to the ionization energy of o-dimethylbenzene and/or m-dimethylbenzene. The ionization potential of ethylbenzene is $8.77 \mathrm{eV}$, so its contribution to the $\mathrm{C}_{8} \mathrm{H}_{10}{ }^{+}$signal cannot be ruled out. The contribution of p-dimethylbenzene may be somewhat significant. The PIE spectra of heavier compounds are more complex due to the presence of many isomers. Even so, the PIE shape provides some hints for identifying isomers. The PIE spectrum of $\mathrm{C}_{9} \mathrm{H}_{12}(120 \mathrm{u})$ shown in Figure 4c indicates a marked onset at $8.5 \mathrm{eV}$ and a tail down to 8.2 $\mathrm{eV}$. This shape may be interpreted as resulting from the contribution of trimethylbenzene isomers, as depicted in the Figure 4c. In addition, the steep rise at $8.8 \mathrm{eV}$ may reflect the contribution of other compounds such as 
isopropylbenzene or possibly propylbenzene (IP $8.7 \mathrm{eV}$ [16] or ethyltoluene isomers (IP not reported in the NIST database).

\section{Semiquantitative Analysis}

From the mass spectra at a fixed wavelength, it is possible to determine the absolute concentration $C_{i}$ of each compound with molecular weight $\mathrm{m}_{\mathrm{i}}$. In fact, the ion signal $S_{i}$ produced by photons of wavelength $\lambda$ can be expressed as

$$
\mathrm{S}_{\mathrm{i}}=\alpha\left(\lambda, \mathrm{m}_{\mathrm{i}}\right) \times \mathrm{s}_{\mathrm{i}}(\lambda) \times \mathrm{C}_{\mathrm{i}}
$$

where $C_{i}$ is the concentration (in ppmv) of the i-th species in the sample, $\sigma_{\mathrm{i}}(\lambda)\left(\mathrm{cm}^{2}\right)$ is the absolute photoionization cross section at wavelength $\lambda$, and $\alpha\left(\lambda, \mathrm{m}_{\mathrm{i}}\right)$ is a factor accounting for the instrumental response, the photon flux, the local density of the molecular beam, and the mass discrimination factor. Using an internal standard, it is possible to cancel out common factors and to retrieve the concentration of each compound, provided that its absolute photoionization cross-section is known. Unfortunately, available data on cross-sections $[3,4,18,19]$ are scarce and/or affected by uncertainties that are sufficiently large to prevent quantitative analysis. Most data are only available at $\lambda=118 \mathrm{~nm}(10.5$ $\mathrm{eV}$ ). This particular wavelength can be obtained from ninth harmonic generation of commercial Nd-YAG lasers and from synchrotron sources.
Figure 5a shows an enlarged view of the mass spectrum of moped exhaust (raw data) at $\lambda=118 \mathrm{~nm}$. Table 2 lists the mass peaks detected in the spectrum of Figure $5 \mathrm{a}$, the onset of each peak in the PIE spectrum, the related chemical formulas, the assignments, and the estimated concentrations. The onset in the PIE spectrum of the detected peaks agrees with the ionization potentials of the various gasoline components [16]. The mass spectrum in Figure 5a does not show any detected peaks at odd $m / z$ values (which could be due to fragments from large hydrocarbons), apart from the peak at $m / z=73$, which is the well-known $\mathrm{C}_{4} \mathrm{H}_{9} \mathrm{O}^{+}$fragment of MTBE [20] (this peak assignment is supported by the corresponding PIE spectrum onset [16]). As a result, the spectrum reported in Figure 5a appears fragment-free. This finding was to be expected because, on one hand, the appearance energy of possible fragments is mostly near and/or higher than the 10.5 ionization energy [16] and, on the other hand, the cooling of the compounds in the molecular beam determines lower abundances of ionized fragments at ionization energies near the appearance energy $[1,2]$.

As shown in Table 2, three classes of compounds (aromatics, olefins, and paraffins) can be identified. According to literature data [18], the photoionization cross-sections of these species are quite similar within each class of compounds. In comparison to the benzene cross-section $\left(\sigma_{\mathrm{b}}=22 \mathrm{Mb}\right)$, the mean values are $\sim 0.1 \times$ $\sigma_{\mathrm{b}}$ for paraffins, $\sim 0.4 \times \sigma_{\mathrm{b}}$ for olefins and ethers, and $\sim 1.0 \times \sigma_{\mathrm{b}}$ for benzene derivatives [18]. It is worth

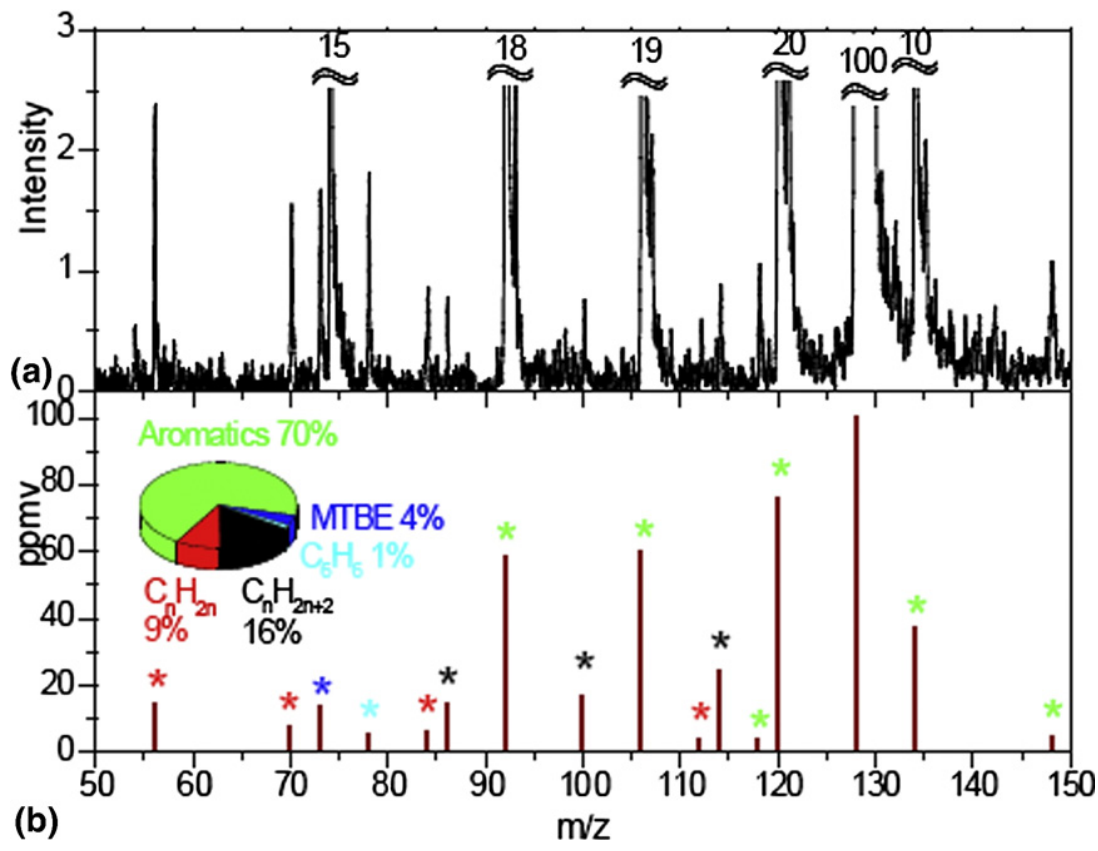

Figure 5. (a) Enlarged view of raw data for a mass spectrum accumulated using 5000 acquisition events at $10.5 \mathrm{eV}$ photon energy. The intensity value of the scaled-off peaks is reported at the top of each peak. (b) The concentration histogram of the spectrum shown in Figure 5a, retrieved as described in the text. The peak corresponding to diethyl ether $(m / z=74)$ was skipped. The vertical scale is the single species concentration (ppmv) in He-diluted exhaust. The inset is the chart of the relative concentration (\%) of classes of compounds found in the moped exhaust gas. The color of the stars shown at the top of the various peaks indicates the class of the compounds as reported in the pie chart. 
Table 2. Mass peaks detected in the spectrum in Figure 5A with the measured PIE spectrum onset and the total concentration of specific compounds

\begin{tabular}{rcllr}
\hline$m / z$ & PIE onset $(\mathrm{eV})$ & Formula & Assigned compounds & Concentration (ppmv) \\
\hline \hline 56 & 9.4 & $\mathrm{C}_{4} \mathrm{H}_{8}$ & 1-Butene & 14 \\
70 & 9 & $\mathrm{C}_{5} \mathrm{H}_{10}$ & 2 -Bentene & 7 \\
73 & 9.6 & $\mathrm{C}_{4} \mathrm{H}_{9} \mathrm{O}^{+}$ & MTBE & 14 \\
78 & 9.2 & $\mathrm{C}_{6} \mathrm{H}_{6}$ & Benzene & 5 \\
84 & 9 & $\mathrm{C}_{6} \mathrm{H}_{12}$ & Hexene & 5 \\
86 & 10 & $\mathrm{C}_{6} \mathrm{H}_{14}$ & Hexane/Methyl pentane & 14 \\
92 & 8.8 & $\mathrm{C}_{7} \mathrm{H}_{8}$ & Toluene & 58 \\
100 & 10 & $\mathrm{C}_{7} \mathrm{H}_{16}$ & Heptane & 16 \\
106 & 8.3 & $\mathrm{C}_{8} \mathrm{H}_{10}$ & Xylenes & 60 \\
112 & 9.2 & $\mathrm{C}_{8} \mathrm{H}_{16}$ & 1 -4-Dimethyl cyclohexane & 4 \\
114 & 9.7 & $\mathrm{C}_{8} \mathrm{H}_{18}$ & Octane/isooctane & 24 \\
118 & 8.4 & $\mathrm{C}_{9} \mathrm{H}_{10}$ & Indane & 3 \\
120 & 8.2 & $\mathrm{C}_{9} \mathrm{H}_{12}$ & Trimethylbenzenes & 76 \\
134 & 8.1 & $\mathrm{C}_{10} \mathrm{H}_{14}$ & Tetramethylbenzenes & 34 \\
148 & 8.4 & $\mathrm{C}_{11} \mathrm{H}_{16}$ & Methylbutylbenzenes & 4 \\
\hline
\end{tabular}

The uncertainty of the concentrations is discussed in the text. The assigned compounds refer to isomers identified by the onset of the PIE spectrum. lonization energy data from references [5] and [16] have been used for the assignments.

noting that the cross-section values reported in different works for a well-characterized compound such as benzene $[4,18,19]$ differ from one another by about $30 \%$. Similar uncertainties affect the (known) cross-sections of other compounds and are expected for the (unknown) cross-sections of important industrial compounds, e.g., the gasoline additives. Given such uncertainties, we approximately quantify (semiquantitative analysis) various classes of substances using these mean values. As the naphthalene absolute photoionization cross-section is not available, we need a preliminary calibration of naphthalene signals, normalized versus benzene, using a calibrated mixture of the two species. Afterward, we normalize the peak intensity of other compounds to that of naphthalene, which is present in all samples at its saturated vapor pressure. Finally, we use the mean values of the photoionization crosssections to scale each peak in the mass spectrum of Figure $5 \mathrm{a}$ and to provide an approximate concentration of each individual component in Figure 5b. Despite the large uncertainties, the general agreement with the VOC speciation data from motorcycle exhaust is quite good [13]. The inset in Figure 5b shows a chart of the mean composition of the moped exhaust. In the cold start condition, when a substantial amount of fuel is unburned, the exhaust composition is expected to be similar to that reported in Table 1. A good agreement is observed for olefins, benzene, and MTBE. The significant discrepancy for the paraffins is likely attributable to the photoionization cross-sections of these compounds, which are much lower than those of the aromatics. In particular, the pentane photoionization cross-section $(0.7 \mathrm{Mb})$ is the lowest among the paraffin class [18]. It is then not surprising that the $\mathrm{C}_{5} \mathrm{H}_{12}{ }^{+}$ion (corresponding to pentane and/or isopentane, which in commercial gasoline constitutes a substantial fraction of the total paraffins) is completely missed or hardly detected in the mass spectrum, as far as the concentration of neutral species is lower than 30-50 ppmv.

\section{Conclusions}

A TOF mass spectrometer was developed using a laser-produced plasma as the VUV photoionization source. Its performance was tested by studying the composition of moped exhaust gas. The detection limit for aromatic compounds was found to be about $1 \mathrm{ppm}$. Substituted benzene derivatives and some olefins were easily detected in the complex gaseous mixture, but paraffins were difficult to detect due to their much lower photoionization cross-sections. Improvements in the overall sensitivity can be achieved with better optimized optical components. The characterization reported here shows two advantages over conventional TOF-MS schemes: (1) soft ionization, achieved using non-coherent VUV photons, avoids fragmentation of the various components present in complex mixtures, thus simplifying sample speciation, and (2) even at moderate/low photon energy resolution, information on the isomeric contribution to the mass peaks can be gained from the PIE spectrum analysis. We also showed that semiquantitative analysis can be performed using the mean values of the absolute photoionization crosssections of classes of compounds. A fully quantitative analysis is expected to be feasible as soon as the ionization potentials and the absolute ionization cross-sections for the species of interest are available. Finally, our findings encourage the use of laserproduced plasmas as table-top and tunable singlephoton ionization sources in TOF mass spectrometry, particularly for comprehensive analysis of complex gaseous mixtures. 


\section{Acknowledgments}

The authors thank Bruno Sgammato for his technical support. T.M.D. acknowledges financial support from Ricerca Spontanea a Tema Libero (Curiosity Driven Research) grant no. 326 of the Italian National Research Council. A.B. is indebted to the continuing mentoring of Professor Antonio D'Alessio.

\section{References}

1. Nir, E.; Hunziker, H. E.; de Vries, M. S. Fragment-Free Mass Spectrometric Analysis with Jet Cooling/VUV Photoionization. Anal. Chem. 1999, 71, 1674-1678.

2. Weitzel, K. M.; Booze, J. A.; Baer, T. Shifts in Photoionization Fragmentation Onset. A Direct Measure of Cooling in a Supersonic Molecular Beam. Chem. Phys. 1991, 150, 263-273.

3. Wang, J.; Yang, B.; Cool, T. A.; Hansen, N.; Kasper, T. Near-Threshold Absolute Photoionization Cross-Sections of Some Reaction Intermediates in Combustion. Int. J. Mass Spectrom. 2008, 269(3), 210-220.

4. Cool, T. A.; Wang, J.; Nakajiama, K.; Taatjes, C. A.; Mclllroy, A. Photoionization Cross Sections for Reaction Intermediates in Hydrocarbon Combustion. Int. J. Mass Spectrom. 2005, 247, 18-27.

5. Wang, J.; Yang, B.; Li, Y.; Tian, Z.; Zhang, T.; Qui, F.; Nakajiama, K. The Tunable VUV Single-Photon Ionization Mass Spectrometry for the Analysis of Individual Components in Gasoline. Int. J. Mass Spectrom. 2007, 263, 30-37.

6. Yang, Z.; Zhang, T.; Pan, Y.; Hong, X.; Tang, Z.; Qi, F. Electrospray/ VUV Single-Photon Ionization Mass spectrometry for the Analysis of Organic Compounds. J. Am. Chem. Soc. Mass Spectrom. 2009, 20(3), $430-434$.

7. Hannaa, S. J.: Campuzano-Jost, P.:Simpson, E. A.; Robb, D. B.; Burak, I. Blades, M. W.; Hepburn, J. W.; Bertram, A. K. A New Broadly Tunable $(7.4-10.2 \mathrm{eV})$ Laser Based VUV Light Source and its First Application to Aerosol Mass Spectrometry. Int. J. Mass Spectrom. 2009, 279, 134-146.
8. Di Palma, T. M.; Borghese, A. Characterization of a UV-VUV Light Source Based on a Gas-Target ns Laser-Produced Plasma. Nucl. Instrum. Methods Phys. Res. B 2007, 254, 193-199.

9. Borghese, A.; Di Palma, T. M. Laser-Plasma Based Vacuum-Ultraviolet Light Source for Tunable Single-Photon Ionization. Appl. Opt. 2007, 46(22), 4948-4953.

10. Prati, M. V.; Costagliola, M. A. Emission of Fine Particles and Organic Compounds from Mopeds. Environ. Eng. Sci. 2009, 26(1), 1-11.

11. Saxer, C. J.; Forss, A. M.; Rudy, C.; Heeb, N. V. Benzene, Toluene and $\mathrm{C}_{2}$-Benzene Emission of a Four-Stroke Motorbikes: Benefit and Risks of the Current TWC Technology. Atmos. Environ. 2006, 40, 6053-6065.

12. Spezzano, P.: Picini, P.: Cataldi, D. Gas and Particle Phase Distribution of Polycyclic Aromatic Hydrocarbons in Two-Stroke, $50-\mathrm{cm}^{3}$ Moped Emission. Atmos. Environ. 2009, 43, 539-545.

13. Tsai, J. H.; Chiang, H. L.; Hsu, Y. C.; Weng, H. C.; Yang, C. Y. The Speciation of Volatile Organic Compounds (VOCs) from Motorcycle Engine Exhaust at Different Driving Modes. Atmos. Environ. 2003, 37 2485-2496.

14. Muhlberger, F.; Zimmermann, R.; Kettrup, A. A Mobile Mass Spectrometer for Comprehensive On-Line Analysis of Trace and Bulk Components of Complex Gas Mixtures: Parallel Application of the Laser-Based Ionization Methods VUV Single-Photon Ionization, Resonant Multiphoton Ionization, and Laser-Induced Electron Impact Ionization. Anal. Chem. 2001, 73(15), 3590-3604.

15. Muhlberger, F.; Wieser, J.; Ulrich, A.; Zimmermann, R. Single Photon Ionization (SPI) via Incoherent VUV-Excimer Light: Robust and Compact Time-of-Fight Mass Spectrometer for On-Line, Real-Time Processes Gas Analysis. Anal. Chem. 2002, 74(15), 3790-3801.

16. http://webbook.nist.gov/chemistry/.

17. Traeger, J. C.; McLoughlin, R. G. A Photoionization Study of the Energetics of the $\mathrm{C}_{7} \mathrm{H}_{7}{ }^{+}$Ion Formed from $\mathrm{C}_{7} \mathrm{H}_{8}$ Precursors. Int. J. Mass Spectrom. Ion Phys. 1978, 27, 319-333.

18. Adam, T.; Zimmermann, R. Determination of Single Photon Ionization Cross Section for Quantitative Analysis of Complex Organic Mixtures. Anal. Bioanal. Chem. 2007, 389, 1941-1951.

19. Kanno, K.; Tonokura, K. Vacuum Ultraviolet Photoionization Mass Spectra and Cross-Sections for Volatile Organic Compounds at $10.5 \mathrm{eV}$. Appl. Spectrosc. 2007, 61(8), 896-902.

20. Butcher, D. J. Vacuum Ultraviolet Radiation for Single-Photoionization Mass Spectrometry: A Review. Microchem. J. 1999, 62, 354-362. 